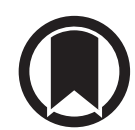

CrossMark

\section{Cumulative effect of smoking on disease burden and multimorbidity in adult-onset asthma}

\author{
To the Editor:
}

Smokers and patients with heavy smoking history have usually been excluded from clinical studies of asthma. Thus, little is known about the impact of lifelong, cumulative tobacco exposure on asthma $[1,2]$. The effect of smoking status (never-, ex- or current smoker) on disease burden of asthma has been more commonly recognised, but the impact of smoking history in pack-years has rarely been evaluated [3-6]. The impact of smoked pack-years has been previously reported only by few studies, showing adverse effects on lung function and asthma control [5,7-10], whereas no significant differences in healthcare use, asthma-related questionnaires or medication use were reported among ex-smokers with severe asthma when patients were categorised based on smoked pack-years [4]. Assessment of pack-years is an easy and usable tool in clinical work, and the intensity of smoking has been proposed to be an even more important factor than plain smoking status [5]. Thus, our aim was to evaluate the impact of cumulative smoking history, i.e. pack-years, on hospitalisations, comorbidities and symptoms in adult-onset asthma, as a part of the Seinäjoki Adult Asthma Study (SAAS).

The Seinäjoki Adult Asthma Study is a prospective, single-centre, 12-year follow-up study of patients with adult-onset asthma. At baseline (years 1999-2002) 257 adults (aged $\geqslant 15$ years) were diagnosed with new-onset asthma by a respiratory physician in Seinäjoki Central Hospital, Finland. Diagnosis was made by following the guidelines, and the study protocol has been previously published [11]. Smokers (ex- or current) were included in the study. After a follow-up of 12 years, 203 (79\%) patients were re-evaluated at a control visit. During the 12 years of follow-up, patients were actively treated for their asthma, following the Finnish Asthma Program guidelines [11]. Data on hospitalisations was retrospectively collected from the patient records of hospitals, primary care, private clinics and occupational care. Structured questionnaires were used to collect information on medication and symptoms. Patients' lifelong smoking history was evaluated based on respiratory nurse interviews, and smoked pack-years (20 cigarettes per day for 1 year) were assessed. Comorbidities were assessed using a structured questionnaire, as previously described [12].

To evaluate the dose-dependent effect of smoking, patients with smoking history (never smokers excluded) were divided into three groups based on smoking history: 1) pack-years 0.1-9.9, 2) pack-years 10-19.9 and $3)$ pack-years $\geqslant 20$. In regression analysis of predictors for hospitalisation, never-smokers were also included. Cross-sectional data from the follow-up visit (years 2012-2013) was used, except when evaluating the use of healthcare resources or medication (oral corticosteroids or antibiotics) during follow-up.

Patients with smoking history exceeding 10 pack-years were older, more obese, and more often males (table 1). Long-acting beta-agonist medication was more often in daily use among patients with $\geqslant 10$ pack-years smoking, but otherwise the medication was similar between the groups. There were no significant differences in daily use of inhaled corticosteroids (ICS) or long-acting muscarinic antagonists, in ICS dose or, surprisingly, in the use of antibiotics or oral corticosteroids (table 1).

The proportion of patients who were hospitalised for any respiratory reason during the 12-year follow-up increased in relation to smoked pack-years, and was the highest in the group with $\geqslant 20$ pack-years of smoking (table 1). Similarly, asthma-related hospitalisations increased in relation to smoked pack-years,

@ERSpublications

Disease burden and multimorbidity in adult-onset asthma increase dose dependently with smoked pack-years http://bit.ly/311627j

Cite this article as: Tommola M, Ilmarinen P, Tuomisto LE, et al. Cumulative effect of smoking on disease burden and multimorbidity in adult-onset asthma. Eur Respir J 2019; 54: 1801580 [https://doi.org/ 10.1183/13993003.01580-2018]. 
TABLE 1 Clinical characteristics, use of medication and effect of cumulative smoking history by pack-year groups

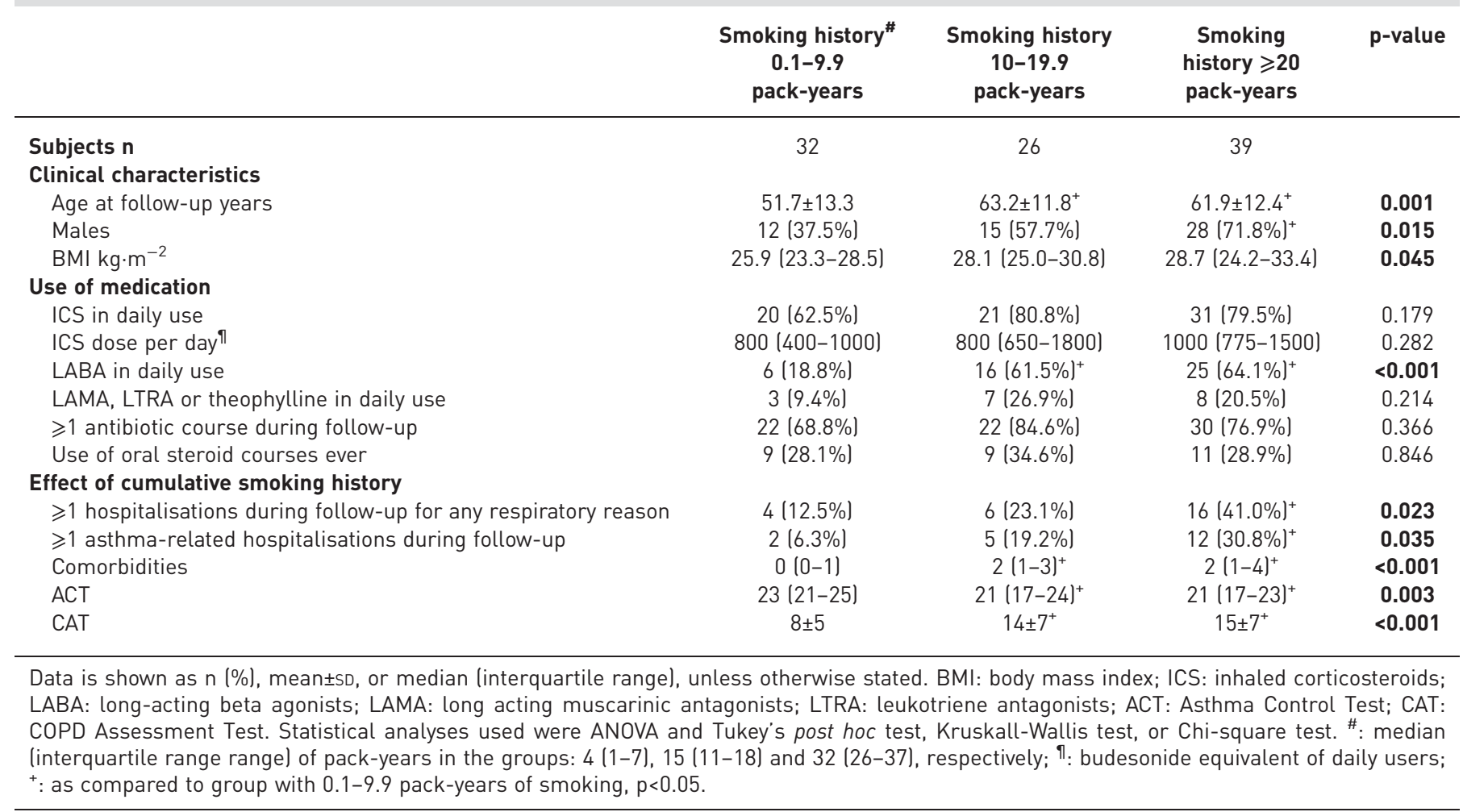

being highest among patients with $\geqslant 20$ pack-years of smoking (table 1). These findings still remained after exclusion of current smokers (data not shown). In addition, the number of pack-years smoked was found to correlate to the number of hospital inpatient periods (Spearman's rho 0.349, $\mathrm{p}<0.001$ ). Patients with smoking history $\geqslant 10$ pack-years had higher number of comorbidities as compared to those with $<10$ pack-years (table 1), and a strong correlation between smoked pack-years and number of comorbidities was found (rho 0.575, $\mathrm{p}<0.001$ ). Patients with light smoking history (0.1-9.9 pack-years) were less symptomatic and had higher Asthma Control Test (ACT) scores and lower COPD Assessment Test (CAT) scores as compared to those with heavier smoking history. CAT scores increased in relation to smoked pack-years (rho $0.452, \mathrm{p}<0.001$ ), being highest among patients with $\geqslant 20$ pack-years (table 1 ).

Predictors of hospitalisation for any respiratory reason were analysed by a multivariable logistic regression model among all patients with the information on pack-years ( $n=193$, never smokers included). Of these, 53 patients were hospitalised during the follow up. Results of the analysis showed that smoking history $\geqslant 20$ pack-years was significantly associated with hospitalisation (OR 2.48, 95\% CI 1.03-5.97; $\mathrm{p}=0.043$ ). Number of comorbidities $\geqslant 2$ was also associated with hospitalisation (OR 4.29, 95\% CI 1.98-9.29; $\mathrm{p}<0.001$ ). In contrast, current smoking (OR $0.46,95 \%$ CI $0.16-1.35 ; \mathrm{p}=0.158$ ), age $>50$ years (OR 0.47 , 95\% CI 0.19-1.13; $\mathrm{p}=0.091$ ), or use of oral corticosteroid courses (OR 1.48, 95\% CI 0.72-3.05; $\mathrm{p}=0.288$ ) were not significantly associated with hospitalisation for respiratory reason. The lack of association between current smoking and hospitalisation may be explained by low number of current smokers.

We showed in this clinical study with long follow-up time that in (current- or ex-) smoking asthmatics, higher numbers of smoked pack-years correlate to more frequent hospitalisations, and a smoking history of $\geqslant 20$ pack-years is a significant predictor of respiratory-related hospitalisation. Previous studies in asthma have suggested association between current smoking and greater risk of unscheduled healthcare visits and hospitalisation [3, 4, 6], but the effect of smoked pack-years has rarely been evaluated. In our study, the relation between increasing rate of respiratory-related hospitalisations during 12 years and higher number of smoked pack-years remained significant even after exclusion of current smokers, which indicates that it may be the cumulative toxic effect of tobacco smoking that increases the risk of adverse health events, not merely current smoking status. This finding emphasises the importance of clinical assessment of smoked pack-years in addition to current smoking status in daily clinical practice. 
In our study, number of comorbidities was found to have a strong positive correlation to the number of smoked pack-years. In addition, having $\geqslant 2$ comorbidities was significantly associated with respiratory-related hospitalisation. Tobacco smoke may cause systemic inflammation [13, 14], which has been recently shown to associate with comorbidities in asthma [12]. Our result on the dose-dependent increase of asthma-related morbidity with smoked pack-years may result from the interplay of toxic effects of tobacco smoke and years of ongoing systemic inflammation.

Increasing number of smoked pack-years was found to correlate with worse symptoms as measured by both ACT and CAT scores. This finding is supported by a previous study reporting more symptoms and worse asthma control questionnaire (ACQ) score in smokers with asthma compared to never-smokers [15]. A correlation between smoked pack-years and ACQ score was also reported [15]. To achieve more reliable assessment of symptoms, we chose both ACT and CAT scores for evaluation, although CAT is more commonly used in chronic obstructive pulmonary disease. Interestingly, the correlation of smoked pack-years to CAT scores was stronger than to ACT scores. The result suggests that CAT score may show the increasing symptoms more sensitively in asthma of smokers, and thus could be considered as a tool for comprehensive evaluation of symptoms.

Taken together, we have shown that in adult-onset asthma, smoked pack-years are associated with more frequent hospitalisations, higher number of comorbidities and more symptoms in a dose-dependent manner. Furthermore, smoking history of $\geqslant 20$ pack-years is significantly associated with a higher risk for respiratory-related hospitalisation. The routine assessment of lifelong smoking history in pack-years should be included in the overall future risk analysis of asthma patients, and early smoking cessation intervention, as well as smoking prevention actions, are crucial.

Minna Tommola $\oplus^{1}$, Pinja Ilmarinen ${ }^{1}$, Leena E. Tuomisto $\oplus^{1}$, Lauri Lehtimäki $\oplus^{2,3}$, Onni Niemelä ${ }^{3,4}$, Pentti Nieminen ${ }^{5}$ and Hannu Kankaanranta $\oplus^{1,3}$

${ }^{1}$ Dept of Respiratory Medicine, Seinäjoki Central Hospital, Seinäjoki, Finland. ${ }^{2}$ Allergy Centre, Tampere University Hospital, Tampere, Finland. ${ }^{3}$ Faculty of Medicine and Health Technology, University of Tampere, Tampere, Finland. ${ }^{4}$ Dept of Laboratory Medicine and Medical Research Unit, Seinäjoki Central Hospital, Seinäjoki, Finland. ${ }^{5}$ Medical Informatics and Statistics Research Group, University of Oulu, Oulu, Finland.

Correspondence: Minna Tommola, Dept of Respiratory Medicine, Seinäjoki Central Hospital, FIN-60220 Seinäjoki, Finland. E-mail: minna.tommola@ksshp.fi

Received: 04 May 2018 | Accepted after revision: 22 April 2019

Acknowledgements: We are grateful to research nurse Aino Sepponen for her input with data management.

This study is registered at ClinicalTrials.gov with identifier number NCT02733016.

Author contributions: M. Tommola, P. Ilmarinen, L.E. Tuomisto, L. Lehtimäki and H. Kankaanranta designed the study and wrote the report with input from the other authors. M. Tommola performed the statistical analyses with help from P. Ilmarinen and P. Nieminen. O. Niemelä contributed to the laboratory analyses. All authors contributed to interpretation of the data. All authors made critical revisions of the manuscript and approved the final version of the manuscript.

Conflict of interest: M. Tommola reports personal fees for lectures and consultancy from Boehringer Ingelheim, personal fees for lectures from AstraZeneca, Filha ry, GlaxoSmithKline and Pfizer, grants from Orion research foundation, outside the submitted work. P. Ilmarinen reports grants from AstraZeneca, personal fees for lectures from MundiPharma, Orion and AstraZeneca, outside the submitted work. L.E. Tuomisto reports non-financial support for attending an international congress from Chiesi, Orion Pharma and TEVA, personal fees for lectures and non-financial support for attending an international congress from Boehringer Ingelheim, personal fees for lectures from AstraZeneca, outside the submitted work. L. Lehtimäki reports personal fees from AstraZeneca, Boehringer Ingelheim, Chiesi, GSK, Mundipharma, Novartis, OrionPharma, Teva and ALK, outside the submitted work. O. Niemelä has nothing to disclose. P. Nieminen has nothing to disclose. H. Kankaanranta reports grants, personal fees for lectures and consulting, costs for attending an international congress from AstraZeneca, personal fees for consulting from Chiesi Pharma AB and Roche, personal fees for lectures and consulting, costs for attending an international congress from Boehringer Ingelheim, personal fees for lectures and consulting from Novartis, personal fees for lectures from Mundipharma and Orion Pharma, outside the submitted work.

Support statement: Supported by the Finnish Anti-Tuberculosis Association Foundation (Helsinki, Finland), Tampere Tuberculosis Foundation (Tampere, Finland), Jalmari and Rauha Ahokas Foundation (Helsinki, Finland), the Research Foundation of the Pulmonary Diseases (Helsinki, Finland), Orion Research Foundation (Espoo, Finland), the Competitive State Research Financing of the Expert Responsibility Area of Tampere University Hospital (Tampere, Finland) and the Medical Research Fund of Seinäjoki Central Hospital (Seinäjoki, Finland). None of the sponsors had any involvement in the planning, execution, drafting or write-up of this study. Funding information for this article has been deposited with the Crossref Funder Registry.

\section{References}

Polosa R, Thomson NC. Smoking and asthma: dangerous liaisons. Eur Respir J 2013; 41: 716-726.

Postma DS, Rabe KF. The Asthma-COPD Overlap Syndrome. N Engl J Med 2015; 373: 1241-1249. 
3 Kauppi P, Kupiainen H, Lindqvist A, et al. Long-term smoking increases the need for acute care among asthma patients: a case control study. BMC Pulm Med 2014; 14: 119.

4 Thomson NC, Chaudhuri R, Heaney LG, et al. Clinical outcomes and inflammatory biomarkers in current smokers and exsmokers with severe asthma. J Allergy Clin Immunol 2013; 131: 1008-1016.

5 Polosa R, Russo C, Caponnetto P, et al. Greater severity of new onset asthma in allergic subjects who smoke: a 10-year longitudinal study. Respir Res 2011; 12: 16.

6 Eisner MD, Iribarren C. The influence of cigarette smoking on adult asthma outcomes. Nicotine Tob Res 2007; 9: 53-56.

7 Tommola M, Ilmarinen P, Tuomisto LE, et al. The effect of smoking on lung function: a clinical study of adult-onset asthma. Eur Respir J 2016; 48: 1298-1306.

8 Hancox RJ, Gray AR, Poulton R, et al. The effect of cigarette smoking on lung function in young adults with asthma. Am J Respir Crit Care Med 2016; 194: 276-284.

9 Tuomisto LE, Ilmarinen P, Niemelä O, et al. A 12-year prognosis of adult-onset asthma: Seinäjoki Adult Asthma Study. Respir Med 2016; 117: 223-229.

10 Westerhof GA, Vollema EM, Weersink EJ, et al. Predictors for the development of progressive severity in new-onset adult asthma. J Allergy Clin Immunol 2014; 134: 1051-1056.

11 Kankaanranta H, Ilmarinen P, Kankaanranta T, et al. Seinäjoki adult asthma study (SAAS): a protocol for a 12-year real-life follow-up study of new-onset asthma diagnosed at adult age and treated in primary and specialised care. NPJ Prim Care Respir Med 2015; 25: 15042.

12 Ilmarinen P, Tuomisto LE, Niemelä O, et al. Comorbidities and elevated IL-6 associate with negative outcome in adult-onset asthma. Eur Respir J 2016; 48: 1052-1062.

13 Ilmarinen P, Tuomisto LE, Kankaanranta H. Phenotypes, risk factors, and mechanisms of adult-onset asthma. Mediators Inflamm 2015; 2015: 514868.

14 Arnson Y, Shoenfeld Y, Amital H. Effects of tobacco smoke on immunity, inflammation and autoimmunity. J Autoimmun 2010; 34: J258-J265.

15 Chaudhuri R, McSharry C, McCoard A, et al. Role of symptoms and lung function in determining asthma control in smokers with asthma. Allergy 2008; 63: 132-135. 